\title{
CORRELATION BETWEEN CORD BLOOD (UCB), 24 HOUR POSTNATAL (24H) BRAIN NATRIURETIC PEPTIDE (BNP) VALUES AND POSTNATAL TRANSITION IN ELECTIVE C/S (ECS) BORN FULL TERM (FT)
}

\author{
P. Srinivasan, P. Jain, L. Cherian, M. Brandler, E. Lavery, E. Koenig, K. Williams \\ Pediatrics, New York Hospital Queens, Flushing, NY, USA
}

Background: Imbalance among hormones and mediators associated with extra-uterine adaptations are linked to the transition failure states such as TTN, a common problem due to decreased lung fluid clearance after ECS. Natriuretic peptides, by decreasing lung fluid production may be important in the fetal adaptive response to extrauterine life. High BNP levels at birth have a crucial regulatory role in the hemodynamic changes associated with extrauterine transition.

\begin{abstract}
Aims: We hypothesized lower UCB BNP levels associated with decreased 24H surge in FT born via ECS will have poor postnatal adaptation. To evaluate BNP levels and determine correlation between UCB and 24H BNP levels with postnatal transition.
\end{abstract}

Methods: IRB approved prospective study,Inclusion:Eligible ECS born FTs Exclusion: presence of risk factors for sepsis, Infants of Diabetic mothers. BNP assays by commercial kit (Triage BNP test). UCB and 24H BNP measured on eligible $(n=66)$ infants. Maternal and infant birth clinical data and incidence of TTN recorded.

Results: Mean GA: 39.1 weeks, Primary vs Repeat ECS: $27 \%$ vs 73 \%, Infant Data: M: F= 47\%: 53\% BW: 3349 grams, DW: 3236 grams, UCB levels are not related to BW and gender. All had significantly increased BNP levels in $24 \mathrm{H}$. None developed TTN, two required NICU admission for LBW/hypothermia and delayed transition respectively. Mean BNP from UCB and $24 \mathrm{H}$ were 5.7 and107.4 pg/ML respectively

Conclusions: FTs born via ECS have appropriate postnatal $24 \mathrm{H}$ surge in BNP and did not show poor neonatal transitional states including TTN in this cohort. Post natal surge is significantly higher in males. 